Supporting Information

\title{
The Cardioprotection Effects of LPTC-5 Involve Mitochondrial Protection and Dynamics
}

Wei Bi, ${ }^{* a}$ Yue Bi, ${ }^{a}$ Pengfei Li $^{a}$ Shanshan Hou, ${ }^{b}$ Xin Yan, ${ }^{b}$ Connor Hensley, ${ }^{b}$ Yanrong Zhang, ${ }^{a}$ Steffen Jockusch, ${ }^{c}$ Thomas D. Legalley, ${ }^{e}$ K. Michael Gibson, ${ }^{d^{*}} \&$ Lanrong Bi $i^{b^{*}}$ 


\section{Experimental Sections:}

General. All the chemicals were purchased from Sigma Aldrich. Unless otherwise stated, all reactions were run under a nitrogen atmosphere $(1 \mathrm{bar})$. The purity $(>97 \%)$ of the intermediates and the final products was confirmed on both TLC (Merck silica gel plates of type 60 F254, 0.25 mm layer thickness) and HPLC (Waters, C18 column 4.6mm x150 mm). NMR spectra were recorded on Bruker Advance 500 spectrometers. FAB-MS was determined by VG-ZAB-MS high resolution GC/MS/DS and HP ES-5989x.

General procedure for the deprotection of the Boc-group: the respective peptide was dissolved in EtOAc and cooled to $0^{\circ} \mathrm{C}$, after which EtOAc $\bullet \mathrm{HCl}(4 \mathrm{M})$ was added and the solution was allowed to warm to room temperature. After stirring at room temperature until starting material was completely consumed (TLC monitoring), the solution was concentrated in vacuo three times to remove residual $\mathrm{HCl}$. The crude residue was titrated with anhydrous ether to give the deprotected peptide.

General Procedure for Removal of Boc of the C-Terminal Component. $0.20 \mathrm{mmol}$ of Bocprotected compound was stirred at room temperature for $3 \mathrm{~h}$ in $2 \mathrm{~mL}$ of hydrogen chloride in ethyl acetate $(4 \mathrm{~mol} / \mathrm{L})$. Solvent was removed by evaporation in vacuo. The residue was dissolved in $10 \mathrm{~mL}$ of ethyl acetate, and the solution evaporated to dryness. The resulting solid was used for the coupling reaction without additional purification.

General procedure for the deprotection of benzyl ester: To a solution of benzyl-protected peptide in $\mathrm{EtOH}, \mathrm{Pd} / \mathrm{C}$ was added and the solution stirred under a hydrogen atmosphere at room temperature. The reaction mixture was filtered and evaporated in vacuo to give the desired product. 

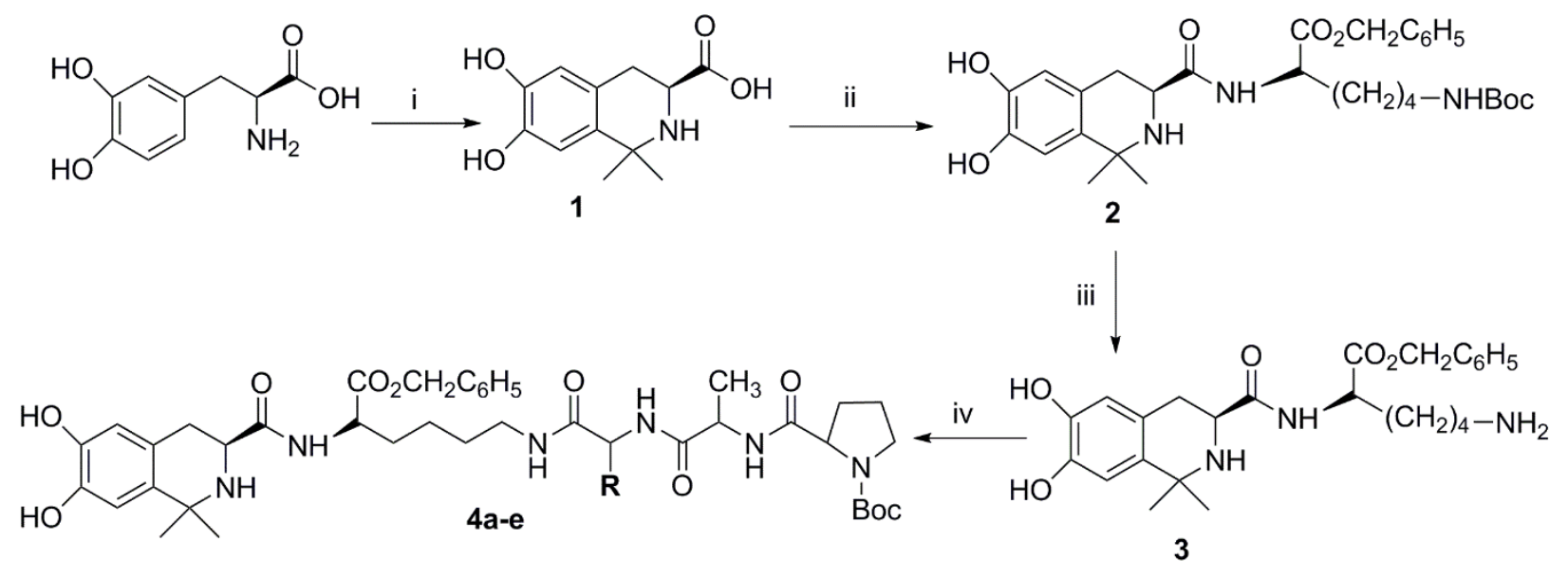<smiles>[R16]c1ccc(CCCCNC(=O)C([R])NC(=O)C(C)NC(=O)C(C)NC(=O)[C@@H]2Cc3cc(O)c(O)cc3C(C)(C)N2)cc1</smiles><smiles>[R]C(NC(=O)C(C)NC(=O)C1CCCN1)C(=O)NCCCCC(NC(=O)[C@@H]1Cc2cc(O)c(O)cc2C(C)(C)N1)C(=O)NC1C(C)(C)CN([O-])CC1(C)C</smiles>

Scheme S1: (i) Acetone, TFA, r.t., 72h; (ii) DCC/HOBt, HCl·Lys(Boc)-OBzl; (iii) EtOAc•HCl (4M); (iv) Boc-Pro-Ala-AA, DCC/HOBt; (v) EtOAc•HCl (4M); (vi) 4-amino-TEMPO.

(S)-6,7-dihydroxy-1,1-dimethyl-1,2,3,4-tetrahydroisoquinoline-3-carboxylic acid (1): To a solution of L-3,4-dihydroxyphenylalanine $(5.0 \mathrm{~g}, 25 \mathrm{mmol})$ in acetone $(250 \mathrm{~mL}), \mathrm{Na}_{2} \mathrm{SO}_{4}(6.0 \mathrm{~g}, 30$ mmol) was added. After stirring at room temperature for 30 minutes, TFA (25 mL) was added. After stirring at room temperature until starting material was completely consumed (TLC monitoring), the solution was filtered and the filtrate was concentrated in vacuo. The resultingresidue was triturated using anhydrous ether $(200 \mathrm{~mL})$ to give the title compound as a 
colorless solid (Yield: 95\%). ESI-MS (m/e): $238[\mathrm{M}+\mathrm{H}]^{+} .{ }^{1} \mathrm{HNMR}\left(\mathrm{DMSO}-d_{6}\right) \delta / \mathrm{ppm}=6.63(\mathrm{~s}$, $1 \mathrm{H}), 6.44(\mathrm{~s}, 1 \mathrm{H}), 3.68(\mathrm{dd}, \mathrm{J}=3.78,11.3 \mathrm{~Hz}, 1 \mathrm{H}), 2.73(d d, J=11.5,15.3 \mathrm{~Hz}, 1 \mathrm{H}), 2.64(\mathrm{~m}, 1 \mathrm{H})$, $1.42(\mathrm{~s}, 3 \mathrm{H}), 1.34(\mathrm{~s}, 3 \mathrm{H})$.

\section{Benzyl N6-(tert-butoxycarbonyl)-N2-((S)-6,7-dihydroxy-1,1-dimethyl-1,2,3,4-tetrahydro-} isoquinoline-3-carbonyl)-L-lysinate (2): To a solution of compound $\mathbf{1}(1.19 \mathrm{~g}, 5.01 \mathrm{mmol})$ in anhydrous DMF (10mL), HOBT (675mg, $5.0 \mathrm{mmol})$ was added. After stirring at $0^{\circ} \mathrm{C}$ for 10 minutes, DCC $(1.20 \mathrm{~g}, 5.83 \mathrm{mmol})$ in anhydrous DMF $(5 \mathrm{~mL})$ was added. Then HCl•Lys(Boc)OBzl• $\mathrm{HCl}(2.83 \mathrm{~g}, 5.53 \mathrm{mmol})$ in anhydrous DMF $(15 \mathrm{~mL})$ was added at $0^{\circ} \mathrm{C}$. The solution was allowed to warm to room temperature and stirred for $12 \mathrm{~h}$. Then NMM (N-methylmorpholine) was added to adjust $\mathrm{pH}$ of the reaction mixture to 9 . After filtration, the filtrate was concentrated in vacuo to remove residual DMF, the residue was then dissolved in EtOAc $(150 \mathrm{~mL})$ and washed with saturated $\mathrm{NaHCO}_{3}$ and brine. The combined organic layers were dried over $\mathrm{MgSO}_{4}$, filtered and evaporated in vacuo to yield the crude product. The crude product was then purified via silica column chromatography (eluent: $\mathrm{CH}_{2} \mathrm{Cl}_{2}: \mathrm{MeOH}=50: 1$ ) to give the title compound (Yield: $59 \%$ )

as a pink powder. ESI-MS (m/e): $556[\mathrm{M}+\mathrm{H}]]^{+} ;{ }^{1} \mathrm{HNMR}\left(\mathrm{DMSO}-d_{6}\right) \delta / \mathrm{ppm}=8.63(\mathrm{~s}, 1 \mathrm{H}), 8.50$ $(\mathrm{s}, 1 \mathrm{H}), 8.17(\mathrm{~d}, \mathrm{~J}=7.5 \mathrm{~Hz}, 1 \mathrm{H}), 7.37(\mathrm{~m}, 5 \mathrm{H}), 6.76(\mathrm{~m}, 1 \mathrm{H}), 6.58(\mathrm{~s}, 1 \mathrm{H}), 6.38(\mathrm{~s}, 1 \mathrm{H}), 5.17(\mathrm{~m}$ 2H), $4.33(\mathrm{~m}, 1 \mathrm{H}), 3.56(\mathrm{~m}, 2 \mathrm{H}), 3.37(\mathrm{~m}, 2 \mathrm{H}), 2.89(\mathrm{~m}, 2 \mathrm{H}), 2.58(\mathrm{~d}, \mathrm{~J}=3.9 \mathrm{~Hz}, 1 \mathrm{H}), 2.28(\mathrm{~m}, 1 \mathrm{H})$, $2.17(\mathrm{~s}, 1 \mathrm{H}), 1.71(\mathrm{~m}, 4 \mathrm{H}), 1.38(\mathrm{~s}, 9 \mathrm{H}), 1.35(\mathrm{~s}, 3 \mathrm{H}), 1.27(\mathrm{~s}, 3 \mathrm{H})$.

\section{Benzyl ((S)-6,7-dihydroxy-1,1-dimethyl-1,2,3,4-tetrahydroisoquinoline-3-carbonyl)-L-}

lysinate (3): following the general procedure for the deprotection of Boc protecting groups, we obtained the title compound. Without further purification, compound $\mathbf{3}$ was directly subjected to coupling reactions. 
General Procedure for Coupling C-Terminal and N-Terminal Components. $0.20 \mathrm{mmol}$ of HOBt and $0.25 \mathrm{mmol}$ of dicyclohexylcarbodiimide (DCC) were added to a solution containing $0.20 \mathrm{mmol}$ of the $\mathrm{N}$-terminal component in $5 \mathrm{~mL}$ of anhydrous $\mathrm{THF}$ at $0{ }^{\circ} \mathrm{C}$. The reaction mixture was stirred at $0{ }^{\circ} \mathrm{C}$ for $24 \mathrm{~h}$. Precipitated dicyclohexyl urea was isolated by filtration. The filtrate was evaporated under reduced pressure, and the residue was triturated with petroleum ether to provide the corresponding ester. $0.20 \mathrm{mmol}$ of the C-terminal component and $0.26 \mathrm{mmol}$ of $\mathrm{N}$ methyl-morpholine were then added to a solution of the preceding ester in $10 \mathrm{~mL}$ of anhydrous THF. The reaction mixture was stirred at room temperature for $24 \mathrm{~h}$. Following evaporation, the residue was dissolved in $50 \mathrm{~mL}$ of ethyl acetate. The solution was washed with $5 \%$ sodium bicarbonate, followed by brine, and the organic phase was separated and dried over anhydrous sodium sulfate. Following filtration and evaporation under reduced pressure, the desired product was obtained by chromatographic purification $\left(\mathrm{CHCl}_{3} / \mathrm{CH}_{3} \mathrm{OH}, 30: 1\right)$.

Boc-Pro-Ala-OBzl: Boc-Pro-OH $(0.2 \mathrm{mmol})$, H-Ala-OBzl $(0.26 \mathrm{mmol})$ was subjected to coupling by following the general procedure described above for the coupling of C-terminal and N-terminal components. This provided a $71 \%$ yield in Boc-Pro-Ala-OBzl as a colorless powder.

Boc-Pro-Ala: Following the general procedure described above for the removal of benzyl ester, Boc-Pro-Ala-OBzl was converted into Boc-Pro-Ala-OH, which was directly used for the coupling reaction without further purification. 
Compound 4a: Following the general procedure described above for the coupling reaction, compound 3 (0.2 mmol) and Boc-Pro-Ala-Ala (0.26 mmol) generated (yield: 47\%) compound 4a as a yellow powder. ESI-MS(m/e): $795[\mathrm{M}+\mathrm{H}]^{+} .{ }^{1} \mathrm{H}$ NMR $\left(\mathrm{DMSO}-d_{6}\right) \delta / \mathrm{ppm}=9.16(\mathrm{~s}, 1 \mathrm{H}), 8.90$ (s, 2H), $8.13(\mathrm{~m}, 1 \mathrm{H}), 7.97(\mathrm{~m}, 1 \mathrm{H}), 7.88(\mathrm{~m}, 1 \mathrm{H}), 7.75(\mathrm{~m}, 1 \mathrm{H}), 7.46-7.33(\mathrm{~m}, 5 \mathrm{H}), 6.68(\mathrm{~s}, 1 \mathrm{H})$ $6.49(\mathrm{~s}, 1 \mathrm{H}), 5.15(\mathrm{~m}, 2 \mathrm{H}), 4.26(\mathrm{~m}, 1 \mathrm{H}), 4.27(\mathrm{~m}, 3 \mathrm{H}), 4.14(\mathrm{~m}, 1 \mathrm{H}), 3.65(\mathrm{~m}, 1 \mathrm{H}), 3.13(\mathrm{~m}, 2 \mathrm{H})$ $2.94(\mathrm{~m}, 2 \mathrm{H}), 2.90-2.93(\mathrm{~m}, 2 \mathrm{H}), 2.13-2.09(\mathrm{~m}, 1 \mathrm{H}), 1.78(\mathrm{~m}, 5 \mathrm{H}), 1.61(\mathrm{~s}, 3 \mathrm{H}), 1.50-1.29(\mathrm{~m}$, 17H), $1.29-1.10(\mathrm{~m}, 7 \mathrm{H})$.

Compound $\mathbf{4 b}$ : Following the general procedure described above for the coupling reaction, compound 3 (0.2 mmol) and Boc-Pro-Ala-Val (0.26 mmol) generated compound $\mathbf{4 b}$ (yield: $40 \%)$ as a yellow powder. 4b: ESI-MS(m/e):823 $[\mathrm{M}+\mathrm{H}]^{+} .{ }^{1} \mathrm{H}$ NMR $\left(\mathrm{DMSO}-d_{6}\right) \delta / \mathrm{ppm}=9.17(\mathrm{~s}, 1 \mathrm{H})$, $8.95(\mathrm{~m}, 2 \mathrm{H}), 8.06(\mathrm{~m}, 2 \mathrm{H}), 7.47(\mathrm{~m}, 5 \mathrm{H}), 6.65(\mathrm{~s}, 1 \mathrm{H}), 6.50(\mathrm{~s}, 1 \mathrm{H}), 5.18(\mathrm{~s}, 1 \mathrm{H}), 4.38-4.29(\mathrm{~m}$, 3H), $4.13(\mathrm{~m}, 3 \mathrm{H}), 3.07-2.90(\mathrm{~m}, 5 \mathrm{H}), 2.77(\mathrm{~m}, 6 \mathrm{H}), 2.06(\mathrm{~s}, 1 \mathrm{H}), 1.94(\mathrm{~m}, 1 \mathrm{H}), 1.80(\mathrm{~m}, 5 \mathrm{H}), 1.64$ (s, 3H), $1.52(\mathrm{~s}, 3 \mathrm{H}), 1.40(\mathrm{~m}, 7 \mathrm{H}), 1.23(\mathrm{~m}, 4 \mathrm{H}), 0.85(\mathrm{~m}, 6 \mathrm{H})$.

Compound 4c: Following the general procedure described above for the coupling reaction, compound $3(0.2 \mathrm{mmol})$ and Boc-Pro-Ala-Phe $(0.26 \mathrm{mmol})$ provided (yield: $37 \%)$ of compound $4 \mathbf{c}$ as a yellow powder. 4c: ESI-MS(m/e): $871[\mathrm{M}+\mathrm{H}]+;{ }^{1} \mathrm{H}$ NMR $\left(\mathrm{DMSO}-d_{6}\right) \delta / \mathrm{ppm}=9.25(\mathrm{~s}$, 1H), $9.10(\mathrm{~m}, 2 \mathrm{H}), 8.97(\mathrm{~s}, 1 \mathrm{H}), 8.02-7.94(\mathrm{~m}, 3 \mathrm{H}), 7.40(\mathrm{~m}, 5 \mathrm{H}), 7.24(\mathrm{~m}, 5 \mathrm{H}), 6.68(\mathrm{~s}, 1 \mathrm{H}), 6.50$ (s, 1H), $5.18(\mathrm{~s}, 2 \mathrm{H}), 4.40(\mathrm{~m}, 3 \mathrm{H}), 4.10(\mathrm{~m}, 2 \mathrm{H}), 3.30(\mathrm{~m}, 4 \mathrm{H}), 2.90(\mathrm{~m}, 2 \mathrm{H}), 2.82(\mathrm{~m}, 5 \mathrm{H}), 1.97$ (m, 2H), $1.76(\mathrm{~m}, 8 \mathrm{H}), 1.54(\mathrm{~m}, 3 \mathrm{H}), 1.35(\mathrm{~m}, 14 \mathrm{H}), 1.17(\mathrm{~m}, 4 \mathrm{H})$. 
Compound 4d: Following the general procedure described above for the coupling reaction, compound 3 (0.2 mmol) and Boc-Pro-Ala-Trp $(0.26 \mathrm{mmol})$ provided (37\% yield) compound $4 \mathbf{d}$ as a yellow powder. 4d: ESI-MS(m/e): $910[\mathrm{M}+\mathrm{H}]^{+} .{ }^{1} \mathrm{H}$ NMR $\left(\mathrm{DMSO}-d_{6}\right) \delta / \mathrm{ppm}=9.53(\mathrm{~s}, 1 \mathrm{H})$, $9.24(\mathrm{~m}, 2 \mathrm{H}), 8.96(\mathrm{~m}, 2 \mathrm{H}), 8.07(\mathrm{~d}, 1 \mathrm{H}, \mathrm{J}=7.2 \mathrm{~Hz}), 7.96(\mathrm{~m}, 1 \mathrm{H}), 7.81(\mathrm{~d}, 1 \mathrm{H}, \mathrm{J}=7.2 \mathrm{~Hz}), 7.56$ (m,1H), $7.40(\mathrm{~m}, 5 \mathrm{H}), 7.36(\mathrm{~d}, 1 \mathrm{H}, \mathrm{J}=4.5 \mathrm{~Hz}), 7.26(\mathrm{~m}, 1 \mathrm{H}), 7.13(\mathrm{t}, 1 \mathrm{H}, \mathrm{J}=4.5 \mathrm{~Hz}), 7.07(\mathrm{t}, 1 \mathrm{H}, \mathrm{J}$ $=4.5 \mathrm{~Hz}), 6.68(\mathrm{~s}, 1 \mathrm{H}), 6.60(\mathrm{~s}, 1 \mathrm{H}), 6.55(\mathrm{~s}, 1 \mathrm{H}), 5.16(\mathrm{~s}, 2 \mathrm{H}), 4.45(\mathrm{~m}, 1 \mathrm{H}), 4.37(\mathrm{~m}, 2 \mathrm{H}), 4.10$ (m, 2H), $3.25(\mathrm{~m}, 1 \mathrm{H}), 3.18(\mathrm{~m}, 5 \mathrm{H}), 2.89(\mathrm{~m}, 1 \mathrm{H}), 1.98(\mathrm{~m}, 2 \mathrm{H}), 1.77-1.72(\mathrm{~m}, 8 \mathrm{H}), 1.33(\mathrm{~s}, 3 \mathrm{H})$ $1.25(\mathrm{~s}, 3 \mathrm{H}), 1.95-1.80(\mathrm{~m}, 9 \mathrm{H}), 1.18(\mathrm{~m}, 4 \mathrm{H})$.

Boc-Pro-Ala-Lys(Z)-OBzl: Following the general procedure described above for the coupling of C-terminal and N-terminal components, $0.2 \mathrm{mmol}$ of Boc-Pro-Ala and $0.2 \mathrm{mmol}$ of $\mathrm{HCl} \cdot \mathrm{Lys}(Z)$ OBzl yielded of Boc-Pro-Ala-Lys(Z)-OBzl as a colorless powder ( $88 \%$ yield). mp: $85-87{ }^{\circ} \mathrm{C}$. FAB-MS $(m / e): 639[\mathrm{M}+\mathrm{H}]^{+} .[\alpha]_{\mathrm{D}}{ }^{20}=-8.6(c=0.2, \mathrm{CHCl} 3) .{ }^{1} \mathrm{H}$ NMR $\left(\mathrm{CDCl}_{3}\right): \delta 8.15(\mathrm{~d}$, $J=6.68 \mathrm{~Hz}, 1 \mathrm{H}), 8.13(\mathrm{~d}, J=6.73 \mathrm{~Hz}, 1 \mathrm{H}), 8.10(\mathrm{t}, J=4.62 \mathrm{~Hz}, 1 \mathrm{H}), 7.36(\mathrm{~d}, J=7.86 \mathrm{~Hz}, 2 \mathrm{H})$, $7.37(\mathrm{~d}, J=7.77 \mathrm{~Hz}, 2 \mathrm{H}), 7.33(\mathrm{t}, J=7.89 \mathrm{~Hz}, 1 \mathrm{H}), 7.33(\mathrm{t}, J=7.79 \mathrm{~Hz}, 1 \mathrm{H}), 7.24(\mathrm{t}, J=7.85 \mathrm{~Hz}$ 2H), $7.23(\mathrm{t}, J=7.77 \mathrm{~Hz}, 2 \mathrm{H}), 4.33(\mathrm{~m}, 1 \mathrm{H}), 5.34(\mathrm{~s}, 2 \mathrm{H}), 5.33(\mathrm{~s}, 2 \mathrm{H}), 4.24(\mathrm{dt}, J=6.73 \mathrm{~Hz}$ $J=4.67 \mathrm{~Hz}, 1 \mathrm{H}), 3.47(\mathrm{t}, J=4.80 \mathrm{~Hz}, 2 \mathrm{H}), 2.96(\mathrm{dt}, J=4.77 \mathrm{~Hz}, J=4.62 \mathrm{~Hz}, 2 \mathrm{H}), 2.24(\mathrm{t}$, $J=4.70 \mathrm{~Hz}, 2 \mathrm{H}), 1.98(\mathrm{~m}, 2 \mathrm{H}), 1.96(\mathrm{dt}, J=4.80 \mathrm{~Hz}, J=4.75 \mathrm{~Hz}, 2 \mathrm{H}), 1.56(\mathrm{~m}, 2 \mathrm{H}), 1.35(\mathrm{~m}$, $2 \mathrm{H}), 1.49(\mathrm{~s}, 3 \mathrm{H}), 1.48(\mathrm{~s}, 9 \mathrm{H})$.

Boc-Pro-Ala-Lys (Z): Boc-Pro-Ala-Lys(Z)-OBzl was subjected to selectively deprotection under mild basic condition to provide Boc-Pro-Ala-Lys(Z), which was directly used for the coupling reaction without further purification. ESI-MS (m/e): $639[\mathrm{M}+\mathrm{H}]^{+}$. 


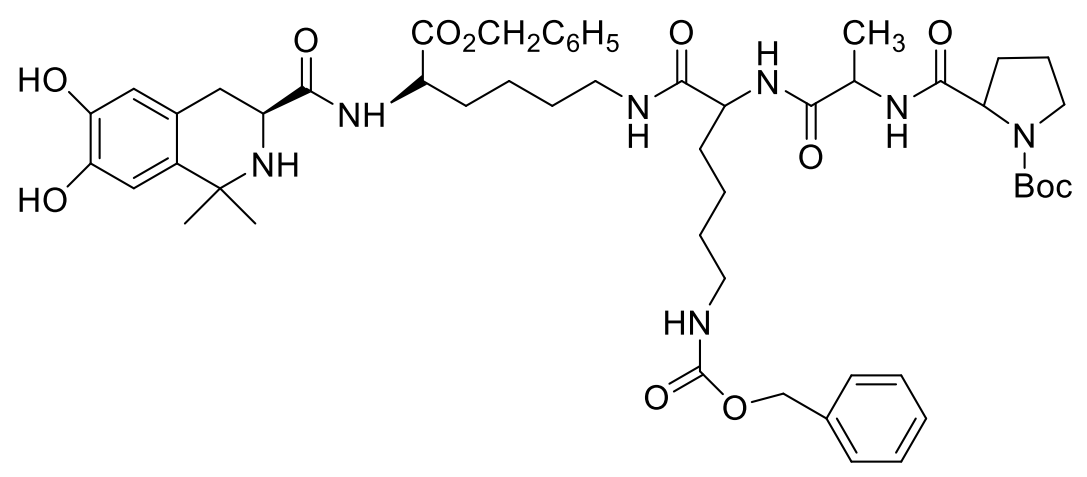

Compound 4e: Following the general procedure described above for the coupling reaction, compound 3 (0.2 mmol) and Boc-Pro-Ala-Lys $(\mathrm{Z})(0.26 \mathrm{mmol})$ provided (39\% yield) compound 4e as a yellow powder. 4e: ESI-MS(m/e): $987[\mathrm{M}+\mathrm{H}]+;{ }^{1} \mathrm{H}$ NMR $\left(\mathrm{DMSO}-d_{6}\right) \delta / \mathrm{ppm}=8.69(\mathrm{~s}$, 1H), $8.57(\mathrm{~s}, 1 \mathrm{H}), 8.18(\mathrm{~m}, 1 \mathrm{H}), 8.05(\mathrm{~m}, 1 \mathrm{H}), 7.79(\mathrm{~m}, 1 \mathrm{H}), 7.69(\mathrm{~m}, 1 \mathrm{H}), 7.35(\mathrm{~m}, 10 \mathrm{H}), 6.57(\mathrm{~s}$ $1 \mathrm{H}), 6.38(\mathrm{~s}, 1 \mathrm{H}), 5.15(\mathrm{~s}, 2 \mathrm{H}), 4.97(\mathrm{~s}, 2 \mathrm{H}), 4.30(\mathrm{~m}, 2 \mathrm{H}), 4.11(\mathrm{~m}, 2 \mathrm{H}), 3.57(\mathrm{~m}, 3 \mathrm{H}), 3.05(\mathrm{~m}$, 4H), $2.66(\mathrm{~m}, 1 \mathrm{H}), 2.43(\mathrm{~m}, 1 \mathrm{H}), 2.28(\mathrm{~m}, 1 \mathrm{H}), 2.18(\mathrm{~s}, 1 \mathrm{H}), 2.07(\mathrm{~m}, 1 \mathrm{H}), 2.04(\mathrm{~s}, 1 \mathrm{H}), 1.80(\mathrm{~m}$, $6 \mathrm{H}), 1.39-1.30(\mathrm{~m}, 18 \mathrm{H}), 1.18(\mathrm{~m}, 10 \mathrm{H})$.

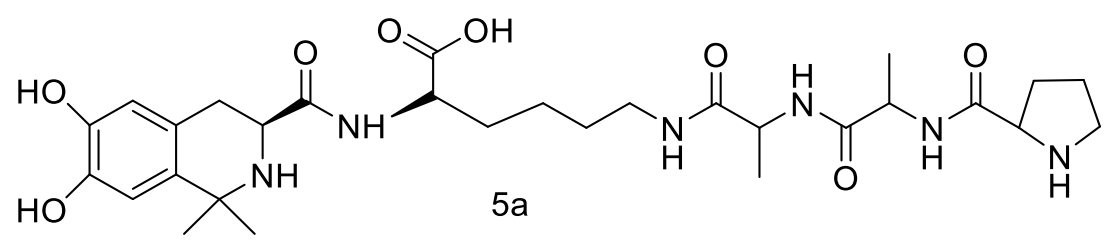

Compound 5a: Following the general procedure described above for the removal of benzyl ester and Boc protecting groups, compound $\mathbf{4 a}$ was converted to 5a. The crude product was then purified via silica column chromatography to give the title compound as a colorless powder. Mp: 207.2$209.4{ }^{\circ} \mathrm{C} ;[\alpha]_{\mathrm{D}}{ }^{25}=-4.5\left(\mathrm{c}=0.25, \mathrm{CH}_{3} \mathrm{OH}\right) ; \operatorname{ESI}-\mathrm{MS}(\mathrm{m} / \mathrm{e}): 630[\mathrm{M}-\mathrm{H}]-{ }^{1} \mathrm{HNMR}\left(\mathrm{D}_{2} \mathrm{O}\right), \delta / \mathrm{ppm}=$ 
$6.80(\mathrm{~s}, 1 \mathrm{H}), 6.70(\mathrm{~s}, 1 \mathrm{H}), 4.37-4.30(\mathrm{~m}, 5 \mathrm{H}), 3.33-3.10(\mathrm{~m}, 6 \mathrm{H}), 1.91(\mathrm{~m}, 4 \mathrm{H}), 1.71(\mathrm{~s}, 3 \mathrm{H}), 1.58$ (s, 3H), $1.49(\mathrm{~m}, 2 \mathrm{H}), 1.35(\mathrm{~m}, 9 \mathrm{H})$.<smiles>CC(NC(=O)C1CCCN1)C(=O)NC(C(=O)NCCCCC(NC(=O)[C@@H]1Cc2cc(O)c(O)cc2C(C)(C)N1)C(=O)O)C(C)C</smiles>

Compound 5b: Following the general procedure described above for the removal of benzyl ester and Boc protecting groups, compound $\mathbf{4 b}$ was converted to $\mathbf{5 b}$. The crude product was then purified via silica column chromatography to give the title compound as a colorless powder. Mp: 195.8-197.9 ${ }^{\circ} \mathrm{C} ;[\alpha]_{\mathrm{D}}{ }^{25}=-3.5\left(\mathrm{c}=0.25, \mathrm{CH}_{3} \mathrm{OH}\right) ; \operatorname{ESI}-\mathrm{MS}(\mathrm{m} / \mathrm{e}): 631.35[\mathrm{M}-\mathrm{H}] .{ }^{1} \mathrm{HNMR}\left(\mathrm{D}_{2} \mathrm{O}\right)$, $\delta / \mathrm{ppm}=8.15(\mathrm{~m}, 1 \mathrm{H}), 6.80(\mathrm{~s}, 1 \mathrm{H}), 6.70(\mathrm{~s}, 1 \mathrm{H}), 4.38-4.30(\mathrm{~m}, 4 \mathrm{H}), 3.91(\mathrm{~m}, 1 \mathrm{H}), 3.37(\mathrm{~m}, 2 \mathrm{H})$ $3.25(\mathrm{~m}, 2 \mathrm{H}), 3.10(\mathrm{~m}, 2 \mathrm{H}), 1.90(\mathrm{~m}, 4 \mathrm{H}), 1.72(\mathrm{~s}, 3 \mathrm{H}), 1.58(\mathrm{~s}, 3 \mathrm{H}), 1.50(\mathrm{~m}, 1 \mathrm{H}), 1.38(\mathrm{~m}, 6 \mathrm{H})$ $1.35(\mathrm{~m}, 2 \mathrm{H}), 0.93(\mathrm{~m}, 6 \mathrm{H})$.

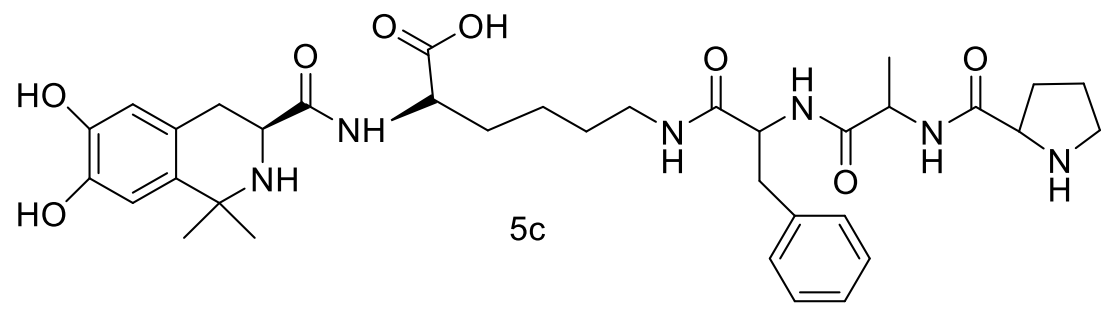

Compound 5c: Following the general procedure described above for the removal of benzyl ester and Boc protecting groups, compound $4 \mathbf{c}$ was converted to $\mathbf{5 c}$. The crude product was then purified via silica column chromatography to give the title compound as a colorless powder. Mp: 175.8$178.2{ }^{\circ} \mathrm{C} ;[\alpha]_{\mathrm{D}}{ }^{25}=-3.3\left(\mathrm{c}=0.25, \mathrm{CH}_{3} \mathrm{OH}\right) ; \operatorname{ESI}-\mathrm{MS}(\mathrm{m} / \mathrm{e}): 679[\mathrm{M}-\mathrm{H}]-{ }^{1} \mathrm{H}$ NMR $\left(\mathrm{D}_{2} \mathrm{O}\right), \delta / \mathrm{ppm}=$ 
$7.27(\mathrm{~m}, 3 \mathrm{H}), 7.15(\mathrm{~m}, 2 \mathrm{H}), 6.78(\mathrm{~s}, 1 \mathrm{H}), 6.67(\mathrm{~s}, 1 \mathrm{H}), 4.40-4.35(\mathrm{~m}, 3 \mathrm{H}), 4.05(\mathrm{~m}, 3 \mathrm{H}), 3.46(\mathrm{~m}$, 1H), $3.40(\mathrm{~m}, 1 \mathrm{H}), 3.30(\mathrm{~m}, 2 \mathrm{H}), 2.18(\mathrm{~m}, 1 \mathrm{H}), 3.10(\mathrm{~m}, 4 \mathrm{H}), 2.97(\mathrm{~m}, 3 \mathrm{H}), 1.92(\mathrm{~m}, 5 \mathrm{H}), 1.75(\mathrm{~s}$, $3 \mathrm{H}), 1.55(\mathrm{~s}, 3 \mathrm{H}), 1.36(\mathrm{~m}, 6 \mathrm{H})$.<smiles>CC(NC(=O)C1CCCN1)C(=O)NC(Cc1c[nH]c2ccccc12)C(=O)NCCCC[C@H](NC(=O)[C@@H]1Cc2cc(O)c(O)cc2C(C)(C)N1)C(=O)O</smiles>

Compound 5d: Following the general procedure described above for the removal of benzyl ester and Boc protecting groups, compound $\mathbf{4 d}$ was converted to $\mathbf{5 d}$. The crude product was then purified via silica column chromatography to give the title compound as a colorless powder. Mp: 205.1-207.9 ${ }^{\circ} \mathrm{C} ;[\alpha]_{\mathrm{D}}{ }^{25}=-1.3\left(\mathrm{c}=0.25, \mathrm{CH}_{3} \mathrm{OH}\right)$; ESI-MS (m/e): $718[\mathrm{M}-\mathrm{H}] \cdot{ }^{-1} \mathrm{H}$ NMR $\left(\mathrm{D}_{2} \mathrm{O}\right)$, $\delta / \mathrm{ppm}=7.51(\mathrm{~d}, J=7.8 \mathrm{~Hz}, 1 \mathrm{H}), 7.42(\mathrm{~d}, J=7.8 \mathrm{~Hz}, 1 \mathrm{H}), 7.13(\mathrm{t}, J=7.2 \mathrm{~Hz}, 1 \mathrm{H}), 7.06(\mathrm{t}, J=7.2 \mathrm{~Hz}$, 1H), $6.76(\mathrm{~s}, 1 \mathrm{H}), 6.65(\mathrm{~s}, 1 \mathrm{H}), 4.40(\mathrm{~m}, 1 \mathrm{H}), 4.37(\mathrm{~m}, 1 \mathrm{H}), 4.28(\mathrm{~m}, 1 \mathrm{H}), 4.20(\mathrm{~m}, 2 \mathrm{H}), 3.25(\mathrm{~m}$, 2H), $3.17(\mathrm{~m}, 3 \mathrm{H}), 3.07(\mathrm{~m}, 3 \mathrm{H}), 1.85(\mathrm{~m}, 1 \mathrm{H}), 1.70(\mathrm{~m}, 4 \mathrm{H}), 1.48(\mathrm{~m}, 3 \mathrm{H}), 1.39(\mathrm{~m}, 2 \mathrm{H}), 1.26(\mathrm{~m}$, $3 \mathrm{H}), 1.25(\mathrm{~m}, 3 \mathrm{H})$.<smiles>CC(NC(=O)C1CCCN1)C(=O)NC(CCCCN)C(=O)NCCCCC(NC(=O)C1Cc2cc(O)c(O)cc2C(C)(C)N1)C(=O)O</smiles>

Compound 5e: Following the general procedure described above for the removal of benzyl ester and Boc protecting groups, compound $\mathbf{4 e}$ was converted to $\mathbf{5 e}$. The crude product was then purified 
via silica column chromatography to give the title compound as a colorless powder. M.p. 185.6$188.1{ }^{\circ} \mathrm{C} ;[\alpha]_{\mathrm{D}}{ }^{25}=-1.8\left(\mathrm{c}=0.25, \mathrm{CH}_{3} \mathrm{OH}\right) ;$ ESI-MS $(\mathrm{m} / \mathrm{e}): 660[\mathrm{M}-\mathrm{H}]^{-1} .{ }^{1} \mathrm{H}$ NMR $\left(\mathrm{D}_{2} \mathrm{O}\right) \delta / \mathrm{ppm}=$ $6.83(\mathrm{~s}, 1 \mathrm{H}), 6.70(\mathrm{~s}, 1 \mathrm{H}), 4.46(\mathrm{~m}, 1 \mathrm{H}), 4.37(\mathrm{~m}, 1 \mathrm{H}), 4.30(\mathrm{~m}, 1 \mathrm{H}), 4.20(\mathrm{~m}, 2 \mathrm{H}), 3.75(\mathrm{~m}, 2 \mathrm{H})$, 3.25-3.15 (m, 8H), $3.10(\mathrm{~m}, 4 \mathrm{H}), 2.96(\mathrm{~m}, 1 \mathrm{H}), 1.95(\mathrm{~m}, 6 \mathrm{H}), 1.76(\mathrm{~m}, 9 \mathrm{H}), 1.60(\mathrm{~m}, 6 \mathrm{H}), 1.52(\mathrm{~m}$, $2 \mathrm{H}), 1.35-1.30(\mathrm{~m}, 16 \mathrm{H}), 1.20(\mathrm{~m}, 2 \mathrm{H})$.

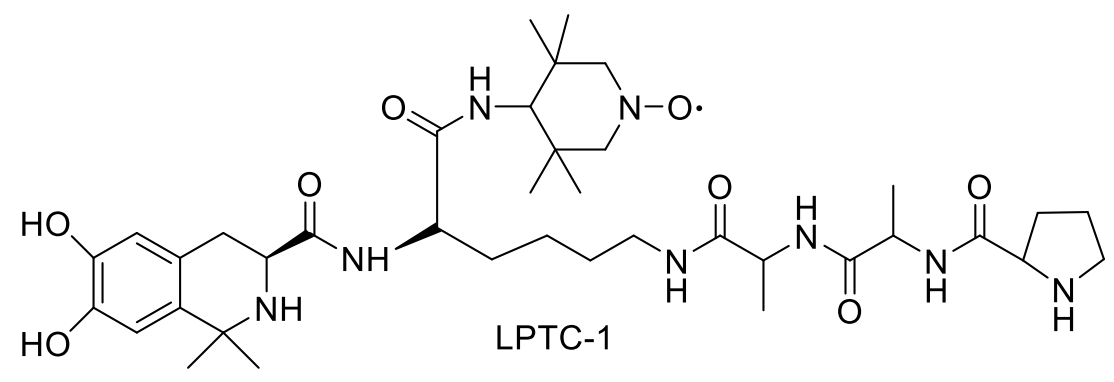

LPTC-1: Following the general procedure described above for the coupling reaction, compound 5a was reacted with 4-amino-TEMPO to give the title compound. Yield: 47\%. ${ }^{1} \mathrm{H}$ NMR (400 $\mathrm{MHz}, \mathrm{CDCl}_{3}$ ) $\delta / \mathrm{ppm}=6.60-6.70$ (brs), 4.55-4.68 (brs), 3.95-4.0 (brs), 2.90-3.20 (brs), 2.50-2.55 (brs), 1.75-1.97 (brs), 1.45-1.55 (brs), 1.25-1.33 (brs). The observed paramagnetic broadening was due to the effect of the free radical from the nitroxide moiety. ${ }^{13} \mathrm{C}$ NMR $\left(100 \mathrm{MHz}\right.$, DMSO- $\left.d_{6}\right)$ $\delta / \mathrm{ppm}=175.1,174.5,170.5,171.5,142.5,142.7,135.7,128.3,111.6,114.5,73.0,60.1,65.0,66.3$ $58.1,52.5,54.2,45.6,41.7,39.2,32.0,31.5,29.4,24.0,31.6,22.3,22.4,18.0 . \mathrm{HR} / \mathrm{MS}[\mathrm{M}+\mathrm{H}]^{+}$ Calcd 757.4612; Found 757.74598. Anal. Calcd for $\mathrm{C}_{38} \mathrm{H}_{61} \mathrm{~N}_{8} \mathrm{O}_{8} \cdot$, C, 60.22; H, 8.11; N, 14.78 . Found: C, 60.15; H, 8.05; N, 14.65 . 


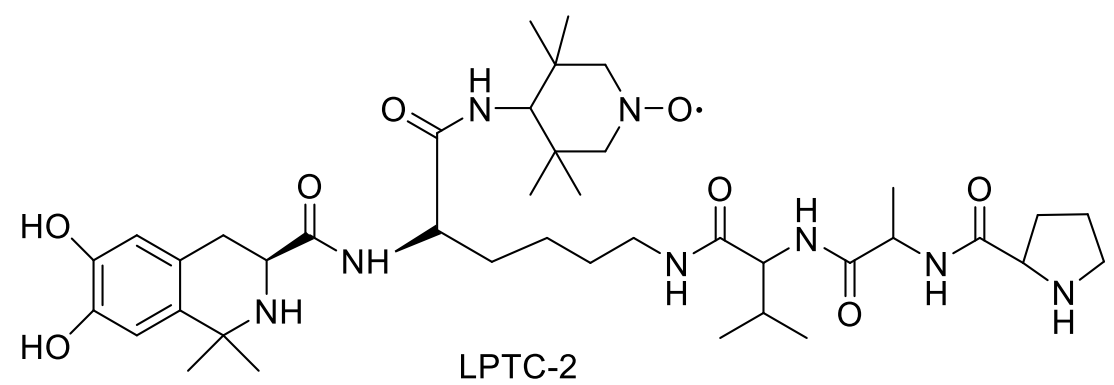

LPTC-2: Following the general procedure described above for the coupling reaction, compound 5b was reacted with 4-amino-TEMPO to give the title compound. Yield: $45 \%$. ${ }^{1} \mathrm{H} \mathrm{NMR} \mathrm{(400} \mathrm{MHz,}$ $\left.\mathrm{CDCl}_{3}\right) \delta / \mathrm{ppm}=6.60-6.68$ (brs), 4.32-4.45 (brs), 4.62-4.64 (brs), 3.95-3.98 (brs), 3.25-3.28 (brs), 2.70-2.80 (brs), 1.91-1.95 (brs), 1.45-1.55 (brs), 1.25-1.30 (brs), 0.94-0.96 (brs). The observed paramagnetic broadening was due to the effect of the free radical from the nitroxide moiety. ${ }^{13} \mathrm{C}$ NMR $\left(100 \mathrm{MHz}, \mathrm{DMSO}-d_{6}\right) \delta / \mathrm{ppm}=175.1,174.5,170.8,171.7,171.1,145.7,142.5,135.8$, $128.5,111.7,114.6,73.1,66.5,65.2,62.5,59.7,58.1,54.4,52.5,45.6,41.7,39.5,32.5,32.1,31.5$, 31.2, 29.5, 22.5, 24.1, 22.5, 18.5, 18.2. HR/MS [M+H] ${ }^{+}$Calcd 785.4925; Found 785.4907. Anal. Calcd for $\mathrm{C}_{40} \mathrm{H}_{65} \mathrm{~N}_{8} \mathrm{O}_{8} \cdot$, C, 61.12; H, 8.34; N, 14.26. Found: C, 61.08; H, 8.29; N, 14.19.

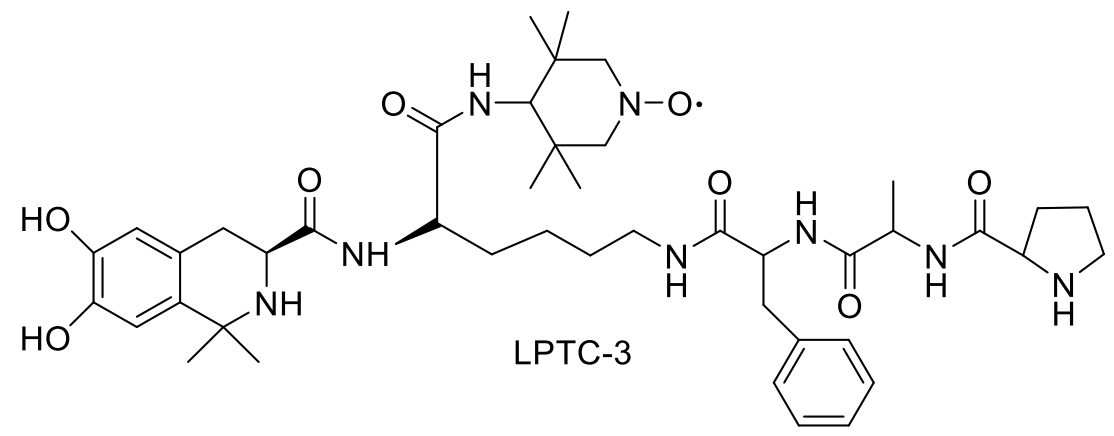

LPTC-3: Following the general procedure described above for the coupling reaction, compound 5c was reacted with 4-amino-TEMPO to give LPTC-3. ${ }^{1} \mathrm{H}$ NMR $\left(400 \mathrm{MHz}, \mathrm{CDCl}_{3}\right) \delta / \mathrm{ppm}=7.13$ 7.20 (brs), 6.60-6.68 (brs), 4.90-4.94 (brs), 4.62-4.64 (brs), 4.42-4.45 (brs), 3.95-3.98 (brs), 3.683.70 (brs), 3.18-3.20 (brs), 3.20-3.29 (brs), 2.78-2.82 (brs), 1.45-1.55 (brs), 1.63-1.78 (brs), 1.25 - 
1.29 (brs), $0.94-0.97$ (brs). The observed paramagnetic broadening was due to the effect of the free radical from the nitroxide moiety. ${ }^{13} \mathrm{C}$ NMR $\left(100 \mathrm{MHz}\right.$, DMSO- $\left.d_{6}\right) \delta / \mathrm{ppm}=171.2,174.5,170.8$, $142.7,142.5,136.7,135.8,128.7,127.8,128.4,126.0,111.7,114.5,73.1,66.4,62.5,65.2,59.8$, $58.9,58.2,53.8,45.7,39.4,37.4,32.4,32.0,31.5,31.0,29.4,25.1,23.8,22.5,22.3,18.1 . \mathrm{HR} / \mathrm{MS}$ $[\mathrm{M}+\mathrm{H}]^{+}$Calcd 834.4925; Found: 834.4901. Anal. Calcd for $\mathrm{C}_{44} \mathrm{H}_{65} \mathrm{~N}_{8} \mathrm{O}_{8}, \mathrm{C}, 63.36 ; \mathrm{H}, 7.86$; N, 13.44. Found: C, 63.3; H, 7.80; N, 13.38 .

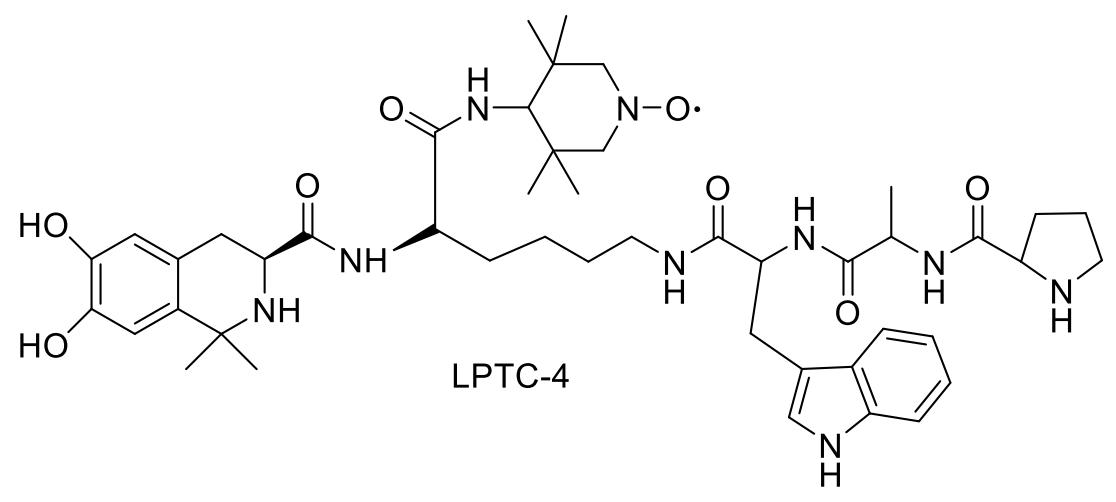

LPTC-4: Following the general procedure described above for the coupling reaction, compound 5d was reacted with 4-amino-TEMPO to give the title compound. Yield: $40 \% .{ }^{1} \mathrm{H}$ NMR (400 MHz, $\left.\mathrm{CDCl}_{3}\right) \delta / \mathrm{ppm}=7.55-7.60$ (brs), 7.33-7.35 (brs), 7.20-7.25 (brs), 6.95-7.10 (brs), 6.60-6.68 (brs), 4.92-4.95 (brs), 4.62-4.65 (brs), 4.42-4.45 (brs), 3.95-4.00 (brs), 2.85-3.20 (brs), 3.20-3.30 (brs), 2.80-3.00(Brs), 2.50-2.55 (brs), 1.95-2.00 (brs), 1.75-1.95 (brs), 1.45-1.55 (brs), 1.25-1.28(brs), 0.95-0.97 (brs). The observed paramagnetic broadening was due to the effect of the free radical from the nitroxide moiety. ${ }^{13} \mathrm{C}$ NMR $\left(100 \mathrm{MHz}, \mathrm{DMSO}-d_{6}\right) \delta / \mathrm{ppm}=175.1,174.6,170.8,171.8$, $142.9,142.7,136.5,135.7,128.5,127.5,123.2,121.8,120.1,118.9,111.7,114.7,111.2,109.8$ $73.1,66.5,65.2,59.8,58.2,52.8,54.2,45.8,41.7,39.5,28.1,32.1,32.0,31.7,31.5,29.5,24.1$, 22.5, 22.3, 18.1. HR/MS [M+H] Calcd 873.5034; Found: 873.5023. Anal. Calcd for $\mathrm{C}_{46} \mathrm{H}_{66} \mathrm{~N}_{9} \mathrm{O}_{8}$, C, 63.28; H, 7.62; N, 14.44. Found: C, 63.20; H, 7.55; N, 14.38 . 


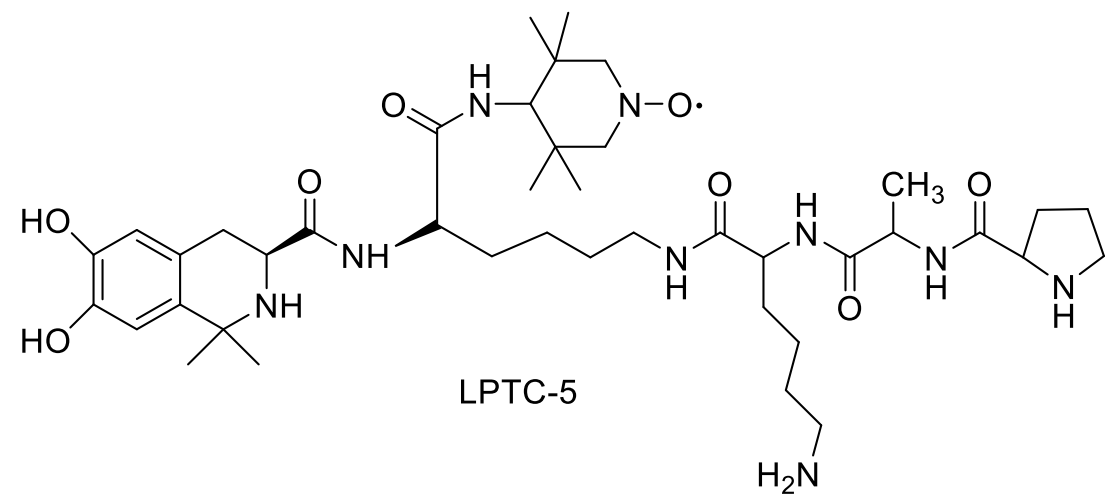

LPTC-5: Following the general procedure described above for the coupling reaction, compound 5e was reacted with 4-amino-TEMPO to give the target compound LPTC-5. Yield: 47\%. ${ }^{1} \mathrm{H}$ NMR $\left(400 \mathrm{MHz}, \mathrm{CDCl}_{3}\right) \delta / \mathrm{ppm}=6.60-6.70$ (brs), 4.45-4.66 (brs), 3.95-3.98 (brs), 3.00-3.30 (brs), 2.682.90 (brs), 2.50-2.55 (brs), 1.95-2.0 (brs), 1.45-1.56 (brs), 1.75-1.95 (brs), 1.25-1.28 (brs), 0.940.96 (brs). The observed paramagnetic broadening was due to the effect of the free radical from the nitroxide moiety. ${ }^{13} \mathrm{C}$ NMR $\left(100 \mathrm{MHz}, \mathrm{DMSO}-d_{6}\right) \delta / \mathrm{ppm}=175.1,174.6,171.8,171.0,170.8$, $142.7,142.9,135.8,128.4,111.7,114.6,60.0,65.1,66.5,73.1,58.2,57.9,54.5,42.3,52.5,45.3$, $41.4,39.5,32.1,32.4,31.7,32.1,31.6,29.5,28.7,24.0,22.4,22.5,18.0 . \mathrm{HR} / \mathrm{MS}[\mathrm{M}+\mathrm{H}]^{+} \mathrm{Calcd}$. 815.5191; Found: 815.5183. Anal. Calcd for $\mathrm{C}_{41} \mathrm{H}_{68} \mathrm{~N}_{9} \mathrm{O}_{8}{ }^{\circ}, \mathrm{C}, 60.42 ; \mathrm{H}, 8.41 ; \mathrm{N}, 15.47$. Found: C, $60.30 ; \mathrm{H}, 8.32 ; \mathrm{N}, 15.38$. 


\section{Biochemical assay}

All animal experiments were performed in compliance with the "Guide for the Care and Use of Laboratory Animals" published by the US National Institutes of Health. The experimental protocol was approved by the Ethics Review Committee for Animal Experimentation of HeBei Medical University.

\section{Cell culture:}

PC12 cells were purchased from the American Type Culture Collection (ATCC) and were grown in DMEM (Dulbecco's modified eagle's medium) supplemented with $10 \%$ of heat inactivated horse serum, $5 \%$ of fetal bovine serum, $1.0 \mathrm{mM}$ sodium pyruvate, $100 \mathrm{U} / \mathrm{mL}$ penicillin, and 100 $\mu \mathrm{g} / \mathrm{mL}$ streptomycin at $37^{\circ} \mathrm{C}$ in $5 \% \mathrm{CO}_{2}$ atmosphere.

Human umbilical vein endothelial cells (HUVECs) were purchased from ATCC and were cultured in endothelial cell medium supplemented with endothelial cell growth supplement, 5\% fetal bovine serum, and penicillin/streptomycin solution in an incubator with $5 \% \mathrm{CO}_{2}$ at $37^{\circ} \mathrm{C}$.

WT MEFs, Mfn KO MEFs, Mfn2 KO MEFs were purchased from ATCC. MEFs cells were cultured in DMEM supplemented with $10 \%$ heat inactivated fetal calf serum, $100 \mathrm{U} / \mathrm{ml}$ penicillin and $100 \mu \mathrm{g} / \mathrm{ml}$ streptomycin in $5 \% \mathrm{CO}_{2}$ at $37^{\circ} \mathrm{C}$. Transfections were performed using Lipofectamine 2000 (Invitrogen) following the manufactures instructions. 


\section{Free radical scavenging assay in $\mathrm{PC12}$ cells}

Free radical scavenging activities were evaluated in PC12 cells by following the previously published procedure. ${ }^{7,9}$ Briefly, PC12 cells were seeded in 96-well plates coated with poly-Llysine at a density of 20,000 cells per well during the exponential phase of growth. After $24 \mathrm{~h}$, fresh media containing TEMPO and LPTCs, were added to each well and were incubated for $1 \mathrm{~h}$. NO• damage was then induced by adding $2 \mathrm{mM}$ of sodium nitroprusside followed by $2 \mathrm{~h}$ of incubation. The media were replaced with fresh media and cells were incubated for $14 \mathrm{~h}$, after which cell survival was measured by a colorimetric assay with MTT according to Mosmann's method. Similarly, $\mathrm{H}_{2} \mathrm{O}_{2}$ damage was induced by $1 \mathrm{~h}$ of incubation with $1 \mathrm{mM} \mathrm{H}_{2} \mathrm{O}_{2}$, and $\bullet \mathrm{OH}$ damage was induced by $1 \mathrm{mM} \mathrm{H} \mathrm{H}_{2} / 30 \mu \mathrm{M}$ Fe (II) followed by $1 \mathrm{~h}$ of incubation.

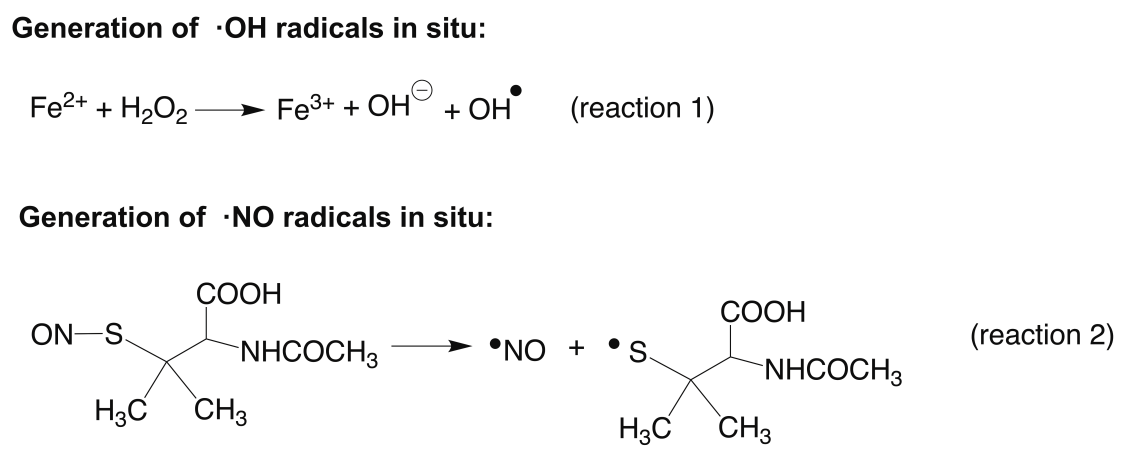

Generation of $\mathrm{O}_{2}^{--\cdot}$ radicals in situ:

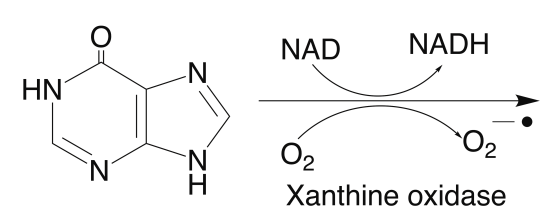

Hypoxanthine

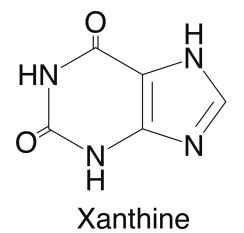

Xanthine

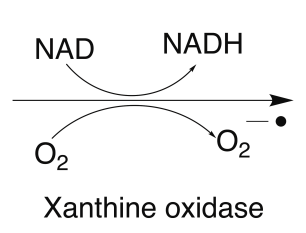<smiles>O=c1[nH]c2nc[nH]c2c(=O)o1</smiles>

(reaction 3)

Figure S1. In the assay, a Fenton-reaction is employed to generate $\cdot \mathrm{OH}$ radicals in situ (Reaction 1). To generate $\cdot \mathrm{NO}$ radicals in situ, the nitric oxide donor $S$-nitroso- $N$-acetyl- $d$,l-penicillamine 
(SNAP) was employed (Reaction 2). For generation of $\mathrm{O}_{2}^{--}$radicals in situ, the reaction of hypoxanthine and xanthine oxidase was employed (Reaction 3).

In vivo antithrombotic activity assays. Test compounds were dissolved in normal saline (NS) prior to use and kept on ice. Male Wistar rats $(250-300 \mathrm{~g})$ were anaesthetized with sodium pentobarbital $(80.0 \mathrm{mg} / \mathrm{kg}$, i.p.) followed by separation of the right carotid artery and left jugular vein. A pre-weighed $6 \mathrm{~cm}$ thread was placed into the middle of a polyethylene tube, filled with sodium heparin (50 IU/mL of NS), and one end inserted into the left jugular vein. Employing the other end of the polyethylene tube, sodium heparin, Normal saline (NS) or test compound in NS was injected and the tube end inserted into the right carotid artery. Blood flow was directed from the right carotid artery toward the left jugular vein via the polyethylene tube for $15 \mathrm{~min}$. The thread was removed, weighed, and the thrombus weight recorded.

\section{Simulated ischemia/reperfusion (s-I/R) protocol}

Simulated I/R was achieved in a flow through chamber as previously described. ${ }^{7,9}$ Briefly, HUVEC cells were washed with phosphate buffer solution (PBS) and incubated in balanced salt solution $\left(116 \mathrm{mmol} / \mathrm{L} \mathrm{NaCl}, 5.4 \mathrm{mmol} / \mathrm{L} \mathrm{KCl}, 0.8 \mathrm{mmol} / \mathrm{L} \mathrm{MgSO}_{4}, 1 \mathrm{mmol} / \mathrm{L} \mathrm{NaH} \mathrm{NaO}_{4}, 0.9\right.$ $\mathrm{mmol} / \mathrm{L} \mathrm{CaCl} 2$, and $10 \mathrm{mg} / \mathrm{L}$ phenol red). The cells were then incubated in a hypoxia chamber (Themo scientific, USA) with a compact gas oxygen controller to maintain oxygen concentration at $1 \%$ by injecting a gas mixture of $94 \% \mathrm{~N}_{2}$ and $5 \% \mathrm{CO}_{2}$ for $2 \mathrm{~h}$. After hypoxia, the cells were transferred back to full culture medium with oxygen. Sham-control cells were incubated in a regular cell culture incubator under normoxic conditions. Cells were incubated with full culture medium containing TEMPO or LPTCs $(30 \mu \mathrm{M})$ under normoxic conditions for $12 \mathrm{~h}$ prior to 
simulated ischemia, respectively. The full culture medium containing drug was then removed. The cells were rinsed once with PBS, and incubated with balanced salt solution containing TEMPO or LPTCs $(30 \mu \mathrm{M})$ during simulated ischemia for $2 \mathrm{~h}$. The balanced salt solution was removed, and the cells incubated with complete culture medium (without drug), under normoxic conditions for $3 h$.

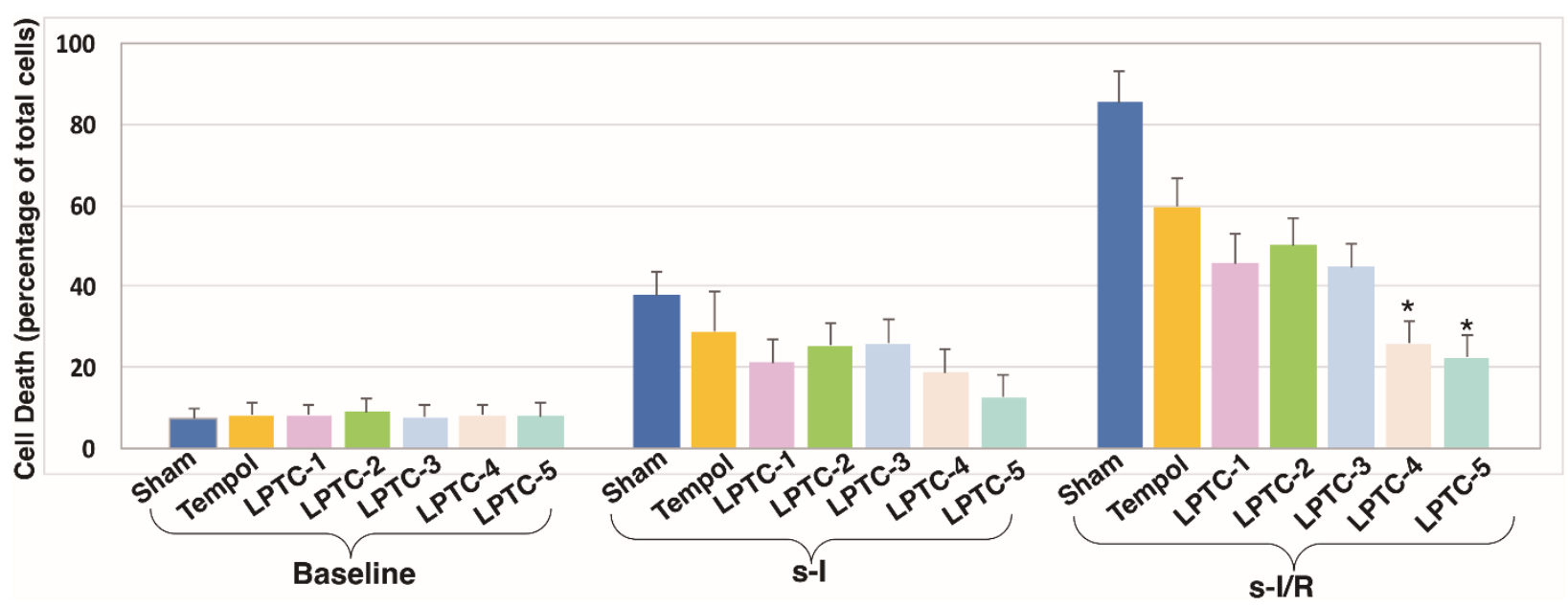

Figure S2. Cell death in simulated ischemia/reperfusion without (sham-control) or with drug (TEMPOL or LPTCs) pretreatment. Error bars indicate standard error of the mean of at least three independent experiments $\left({ }^{*} \mathrm{p}<0.05\right.$ compared to sham control). s-I: simulated ischemia; $\mathrm{s}-\mathrm{I} / \mathrm{R}$ : simulated ischemia and reperfusion. Dose of all of the test compounds ( $(30 \mu \mathrm{M})$. 


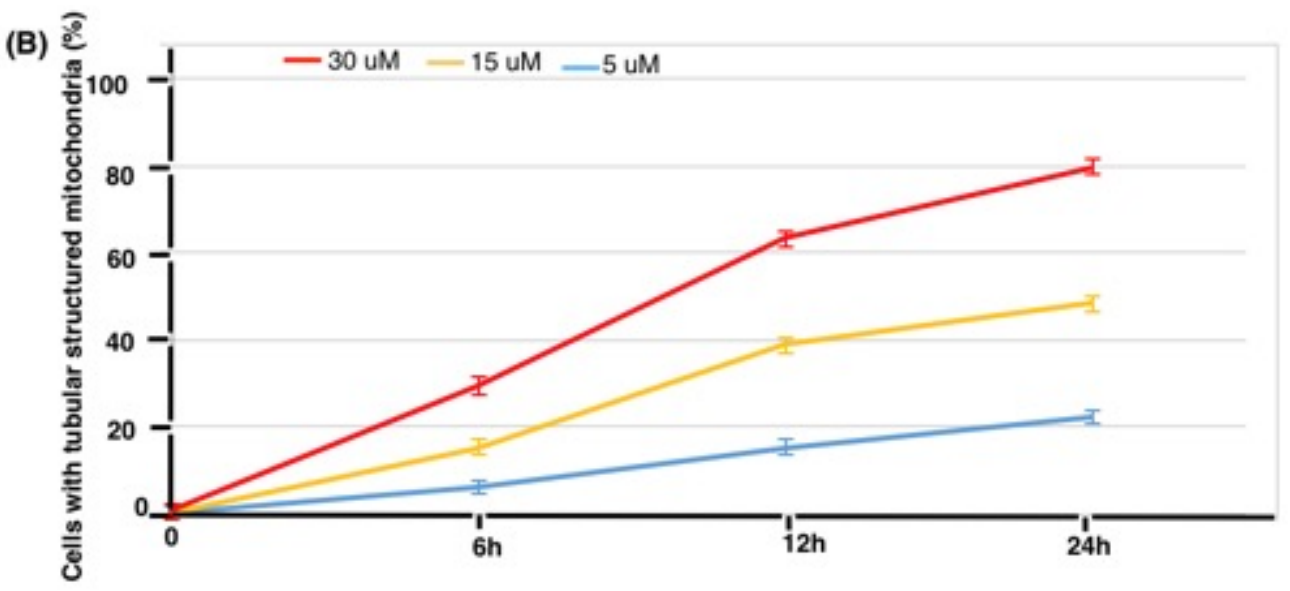

Figure S3. Quantification of cells with tubular structured mitochondria at the indicated time points following incubation with different concentrations $(5,15$ and $30 \mu \mathrm{M})$ of LPTC-5. Data represent the mean $\pm \mathrm{SD}$ of three independent experiments, each with $>100$ cells counted per condition.

Ischemia-reperfusion animal model preparation: Male Wistar rats weighing 250-300 g underwent myocardial ischemia by a temporary occlusion of the left coronary artery as previously $^{7,9}$ described. Briefly, rats were anaesthetized with sodium pentobarbital $(80.0 \mathrm{mg} / \mathrm{kg}$, ip) and placed on an operating table. Polyethylene catheters were inserted into the common femoral artery for the measurement of blood pressure and heart rate, and from the internal carotid artery into the left ventricle for measurement of LVDP (left ventricular developed pressure) and $\mathrm{dP} / \mathrm{dt}_{\max }$ (the maximal rate of rise of left ventricular pressue). After tracheotomy, the animals were ventilated with room air using a small rodent respirator. The chest was opened and the ribs were gently spread. The heart was quickly expressed out of the thoracic cavity, inverted and a $4-0$ silk ligature was placed under the left coronary artery. The heart was repositioned in the chest and the animal was allowed to recover for $15 \mathrm{~min}$. A small plastic snare was threaded through the ligature and placed in contact with the heart. Tightening the ligature could occlude the artery and reperfusion was achieved by releasing the tension applied to the ligature ( $/ \mathrm{R}$ groups). Sham $\mathrm{I} / \mathrm{R}$ 
animals underwent the above described surgical procedures, apart from the fact that the $4-0$ silk, passing around the left coronary artery, was not tied (Sham I/R group). The coronary artery was occluded for 30 min followed by 120 min reperfusion and the animals were randomized in the following groups: Group I/R + saline $(\mathrm{n}=6)$ : animals were infused with $0.9 \% \mathrm{NaCl}$ before coronary occlusion. For the remaining three groups, the drugs were given at the onset of, and continued throughout reperfusion. Group I/R + L-DOPA $(n=6)$ received a continuous L-DOPA infusion $(10 \mathrm{mg} / \mathrm{kg})$. Group I/R + TEMPOL ( $\mathrm{n}=6)$ : received a continuous TEMPOL infusion $(10$ $\mathrm{mg} / \mathrm{kg}$ ); Group I/R + LPTC-5 ( $\mathrm{n}=6)$ received a continuous infusion of LPTC-5 $(10 \mathrm{mg} / \mathrm{kg})$. 
Table S1. Effect of test compounds upon cardiac function

\begin{tabular}{|l|l|l|l|l|}
\hline Groups & Mins & $\begin{array}{l}\text { LVDP } \\
(\mathrm{mmHg})\end{array}$ & $\begin{array}{l}\text { Heart Rate } \\
(\text { beat } / \mathrm{min})\end{array}$ & $\begin{array}{l}\mathrm{dP} / \mathrm{dt}_{\max } \\
(\mathrm{mmHg} / \mathrm{s})\end{array}$ \\
\hline Sham-control & Baseline & $105 \pm 2$ & $295 \pm 30$ & $3207 \pm 105$ \\
\hline Saline & $\mathrm{I}_{30} / \mathrm{R}_{120}$ & $68 \pm 4$ & $245 \pm 36$ & $1597 \pm 124$ \\
\hline L-DOPA & $\mathrm{I}_{30} / \mathrm{R}_{120}$ & $85 \pm 2$ & $262 \pm 20$ & $1795 \pm 85$ \\
\hline TEMPOL & $\mathrm{I}_{30} / \mathrm{R}_{120}$ & $79 \pm 4$ & $258 \pm 34$ & $1708 \pm 101$ \\
\hline LPTC-5 & $\mathrm{I}_{30} / \mathrm{R}_{120}$ & $94 \pm 3$ & $289 \pm 19$ & $2130 \pm 77$ \\
\hline
\end{tabular}

Table S2. Effect of test compounds on size of myocardial infarct

\begin{tabular}{|c|c|}
\hline Groups & Infarct size/Area at risk (\%) \\
\hline I/R+ Saline & $48.5 \pm 4.2$ \\
\hline I/R + L-DOPA & $30.5 \pm 2.7$ \\
\hline I/R + TEMPOL & $35.7 \pm 4.3$ \\
\hline I/R + LPTC-5 & $15.2 \pm 1.8$ \\
\hline
\end{tabular}

Histological analysis: The frozen tissue sections $(4-5 \mu \mathrm{m})$ were cut using a cryostat microtome (Leica CM1850 UV clinical cryostat) at $-30{ }^{\circ} \mathrm{C}$, stained with hematoxylin-eosin, modified 
trichrome, or NADH, and then examined under light phase (Olympus-BX51). Histopathology evaluation studies were performed in a double-blind fashion.

Evaluation of infarct size: Infarct size was estimated by injection of $1 \%$ triphenyltetrazolium into hearts at the end of the reperfusion. The coronary artery was again briefly occluded, after which $1 \%$ Evans blue solution was immediately infused to delineate the ischemic area at risk. Area at risk and infarct size were determined using the NIH software image.

Statistical analysis: A two-way ANOVA followed by Scheffé's test was initially applied using the Origin Program to test for differences between groups. If differences were observed, the values were compared using Student's $t$-test for paired data. The values were expressed as means $\pm \mathrm{SE}$. The results were considered significant if $\mathrm{p}$ was $<0.05$. 